\title{
Test-Retest Reliability and Construct Validity of Two-Minute Walk Test in Children and Adolescents with Cerebral Palsy
}

\author{
Asfarina ZANUDIN ${ }^{1, *}$, Yen Yun KHONG ${ }^{1}$, \\ Ling Fong CHONG ${ }^{1}$ and Nor Azizah MOHAMAD ${ }^{2}$
}

${ }^{I}$ Physiotherapy Programme, Centre for Rehabilitation and Special Needs Studies, Faculty of Health Sciences, Universiti Kebangsaan Malaysia, Kuala Lumpur, Malaysia ${ }^{2}$ Physiotherapy Unit, Department of Rehabilitation, UKM Specialist Children's Hospital, Cheras 56000, Kuala Lumpur, Malaysia

("Corresponding author's e-mail: asfarina.zanudin@ukm.edu.my)

Received: 3 March 2020, Revised: 1 March 2021, Accepted: 2 March 2021

\begin{abstract}
This study explored the test-retest reliability and construct validity of a two-minute walk test in children and adolescents with CP Gross Motor Function Classification System (GMFCS) levels I, II, and III. Twenty-six participants with CP (age $11.7 \pm 3.9$ years) were recruited and were assessed twice with the two-minute walk test to determine the test-retest reliability (interval 2 weeks). Minimal detectable changes (MDC95) were calculated. Construct validity was established by comparing the results of the two- and six-minute walk tests during the first assessment. The test-retest reliability was excellent, with intra-class correlation (ICC) $=0.98$ for all participants. The (MDC95) for all participants was $12.7 \mathrm{~m}$. The distance covered by children and adolescents with CP in the two-minute walk test had high correlation ( $\mathrm{r}$ $=0.9, p<0.01)$ with distance covered in the six-minute walk test. The two-minute walk test is a valid and reliable outcome measure to assess walking performance in children and adolescents with $\mathrm{CP}$.
\end{abstract}

Keywords: Psychometric, Validity, Reliability, Two-minute walk test, Young people, Cerebral palsy

\section{Introduction}

Cerebral palsy (CP) is a group of permanent disorders of the development of movement and posture, causing activity limitation, that are attributed to non-progressive disturbance occurring in the developing infant brain [1]; $\mathrm{CP}$ is often associated with premature and low birth weight survivors [2,3]. Children with CP commonly present with spasticity, lower limb flexor muscle group weakness [4], and reduction in selective motor control [5,6], which affect functional and walking ability over time [7]. Children with $\mathrm{CP}$ who are ambulant either with or without the assistive mobility aids will show some degree of pathological or abnormal gait [8]. In terms of functional ability, a five-level classification system, the Gross Motor Function Classification System (GMFCS), has been used to determine which level best represents a child's or youth's abilities [9].

Evaluating walking performance in people with $\mathrm{CP}$ in a clinical setting requires quick, simple, and psychometrically-sound testing to best represent their functional ability and to ensure the effectiveness of physiotherapy. The recommended test to assess walking performance is the six-minute walk, which examines the functional status of a patient, as most of the activities of daily living are performed at submaximal levels of exercise [10-12]. The six-minute walk test has been found to be reliable, with ICC $=0.80$ [13] for children with CP GMFCS levels I and II and ICCs $>0.90$ in GMFCS subgroups [14]. The minimum detectable change (MDC), according to GMFCS, were reported to be $61.9 \mathrm{~m}$ for those with level $1,64.0 \mathrm{~m}$ for level II, and $47.4 \mathrm{~m}$ for level III [14]. As individuals with muscle weakness, reduced 
http://wjst.wu.ac.th

exercise tolerance, or short attention span and who may have difficulties in completing the six-minute walk test, the two-minute walk test may be an alternative [15]. Pin and Choi (2018) reported that the twominute walk test may be more feasible than the six-minute walk test, as it is of shorter duration and requires less energy from the participants [16]. ICCs $>0.80$ have been reported for the intra and interrater reliability of the two-minute walk test in children with and without neuromuscular disorders aged 6 12 years [16]. In addition, the MDCs were revealed to be $15.7 \mathrm{~m}$ for children walking with aids and $16.6 \mathrm{~m}$ for those walking independently [16]. Although the two-minute walk test showed high within day testretest reliability in the previous study [16], to our knowledge, there has been no published report on its reliability and validity in children and adolescents with CP classified as GMFCS levels I, II, and III.

Thus, the main aim of this study was to determine the test-retest reliability and construct validity of the two-minute walk test among children and adolescents with CP GMFCS subgroups (levels I - III). The secondary aim was to establish the MDCs in the total sample and to determine the correlation between 2MWT and GMFCS levels.

\section{Materials and methods}

\section{Study design}

This was a cross-sectional study. A list of potential participants were identified from the Universiti Kebangsaan Malaysia Medical Centre (UKMMC) hospital database.

\section{Subjects}

Participants were included in this study if they were diagnosed with CP, aged between 7 - 16 years, able to ambulate with or without walking aids, and able to follow basic commands. Participants were excluded if they had had a botulinum toxin injection in the last 3 months, had undergone surgical procedure on a lower extremity in the last 6 months, and had any congenital heart disease that affected their ambulation. All identified participants and care givers were provided with written and verbal information about this study during their visit to the hospital. Participants or care givers who agreed to participate in this study signed an informed consent form prior to the walk test. Ethical approval was obtained from the Research Ethics Committee UKMMC, Kuala Lumpur, Malaysia (NN - 2018 - 113).

\section{Procedures}

This study followed standard guidelines and protocols for the two- and six-minute walk tests described by Pin (2014) and the American Thoracic Society Committee, respectively [17,18]. Trained physiotherapy students administered the tests with training sessions of the test's protocols that were performed prior to data collection. During the tests, each participant wore shoes and sat on a wooden chair with their back and arms supported. The participants were asked not to engage in vigorous activity two hours prior to the test. Prior to the assessments, the participants' heart rate, blood pressure, and oxygen saturation $\left(\mathrm{SPO}_{2}\right)$ were taken. The Borg scale was measured before and after the tests for each participant [19]. The Borg scale was used to monitor and guide participants regarding the level of exertion during the walk tests. During the first assessment, each participant was instructed to walk (not run or jog) for two and six minutes on a 15-meter long walkway with an adequate break (10 - 15 min) given between the two walk tests. Baseline resting heart rate should have been achieved before starting the next walk test. The start and end of the walkway were marked with brightly coloured tape. The participants were allowed to rest or stop any time during the test. Verbal encouragement was not given to the participants. The same procedure for the two-minute walk test was repeated after two weeks with different data collection sheets used to prevent bias from the previous results. A two week interval was chosen in view of no changes in walking performance to be expected, as no intervention took place during this period. During the 2 week interval, participants continued their daily living acivities with no physiotherapy sessions. 
http://wjst.wu.ac.th

\section{Statistical analysis}

Data were analysed using IBM SPSS version 23.0. Intra-class correlation coefficient $\left(\mathrm{ICC}_{2,1}\right)$ was used as a parameter to report the test-retest reliability [20]. ICC value $<0.5$ was considered poor, between 0.5 and 0.75 moderate, between 0.75 and 0.9 good, and $>0.90$ excellent [21]. Standard error of measurement $(\mathrm{SEM})$ and minimal detectable changes $\left(\mathrm{MDC}_{95}\right)$ were also calculated. SEM was calculated using the formula $\mathrm{SEM}=\mathrm{SD} *(\sqrt{ } 1-\mathrm{ICC})$, where $\mathrm{SD}$ is the standard deviation of the baseline scores. The $\mathrm{MDC}_{95}$ was calculated at a $95 \% \mathrm{CI}$, where $\left.\mathrm{MDC}_{95}=1.96 * \sqrt{ } 2 * \mathrm{SEM}\right)$ [22]. To explore the mean differences over time (between assessment 1 and 2) and interaction between group and time, mixed model ANOVA test were conducted. To evaluate the construct validity between the two- and six-minute walk tests and correlation between the 2MWT and GMFCS levels, Pearson's correlation coefficients ( $r$ ) were calculated, with $\mathrm{r}<0.30$ regarded as weak, $0.30-0.59$ as moderate, and $\geq 0.60$ as strong [23]. The significance level was set at $p<0.05$. A Bland and Altman plot was provided to give a visual representation of size and range differences between the $x$ (mean of the two-minute walk test taken on two assessments) and $y$ (difference between the two assessments) values [24].

\section{Results and discussion}

A total of 31 children and adolescents with $\mathrm{CP}$ met the inclusion criteria; however, only 26 of them were included in this study. Two of the participants were unable to participate in this study due to anxiety problems prior to the test. Another three participants were unable to participate due to time constraints. For test-retest reliability, six participants were unable to repeat the assessment due to transportation and unrelated medical problems, hence, only 20 participants were included for the reliability analysis. Table 1 shows the demographic data of the participants. The mean age of participants in this study was $11.7 \pm 3.9$ years. Table 2 shows the mean distances covered in both assessments. There was no significant difference between the mean walking distance in the first and second assessments $(p=0.45)$. In addition, there was no significant interaction between group and time with $p=0.78$. The result for the 2MWT testretest was excellent, with $\mathrm{ICC}=0.98$, and the $\mathrm{MDC}_{95}$ were calculated as $12.7 \mathrm{~m}$ for all participants.

Table 1 Demographic data of participants in this study $(n=26)$.

\begin{tabular}{ll}
\hline Characteristics & Frequency (\%) \\
\hline Gender & $13(50.0)$ \\
Male & $13(50.0)$ \\
Female & $15(57.7)$ \\
Age & $11(42.3)$ \\
Children $(7-12)$ & \\
Adolescents (13-16) & $14(53.8)$ \\
GMFCS levels & $6(23.1)$ \\
I & $6(23.1)$ \\
II & \\
III & $14(53.8)$ \\
Type of CP & $12(46.2)$ \\
Spastic diplegia & \\
Spastic hemiplegia & $6(23.0)$ \\
Type of ambulation aid & \\
Rollator
\end{tabular}

GMFCS $=$ Gross Motor Classification Function System 
http://wjst.wu.ac.th

Table 2 Mean distance covered for both assessments of two-min walk test $(n=20)$.

\begin{tabular}{lcc}
\hline Participants & $\begin{array}{c}\text { Mean distance assessment } \\
(\mathbf{1} \text { m) }\end{array}$ & $\begin{array}{c}\text { Mean distance assessment } \\
\mathbf{( 2 ~ m ) ~}\end{array}$ \\
\hline Participants with CP (n=20) & $91.03(32.30)$ & $93.58(34.24)$ \\
Participants with CP GMFCS level I $(\mathrm{n}=11)$ & $107.87(20.87)$ & $111.00(21.78)$ \\
Participants with CP GMFCS level II $(\mathrm{n}=5)$ & $93.37(18.64)$ & $95.20(21.59)$ \\
Participants with CP GMFCS level III $(\mathrm{n}=4)$ & $41.78(20.32)$ & $41.28(19.58)$
\end{tabular}

GMFCS $=$ Gross Motor Classification Function System

Paired t-test results:

All participants $p=0.45$

Participants with CP GMFCS level I $p=0.4$

Participants with CP GMFCS level II $p=0.5$

Participants with CP GMFCS level III $p=0.7$

Figure 1 demonstrates the relationship between the means of the two distances covered in the twominute walk test taken on two assessments ( $x$-axis) against the difference between the two assessments $(y$ axis). Figure 1 suggested that the mean distance increased by approximately $2.6 \mathrm{~m}$ in the second assessment. This indicates that the participants tended to walk slightly faster in the second assessment. The magnitude of this difference is unlikely to be of great clinical significance.

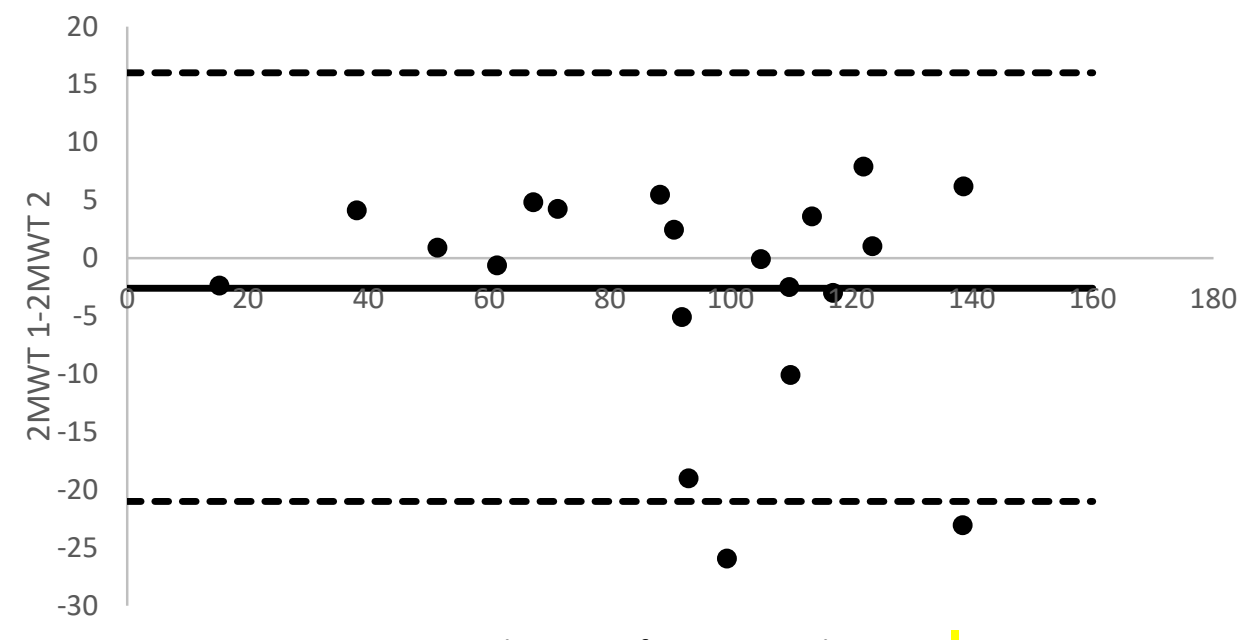

Means distance of 2MWT1 and 2MWT2

Figure 1 Plot of the difference between distances in two assessments against the means of distance for both assessments. The mean difference between distances bisects the y-axis, and lower lines represent the $95 \%$ limits of agreement.

2MWT1 - two-minute walk test in the first assessment

2MWT2 - two-minute walk test in the second assessment 
http://wjst.wu.ac.th

The mean distance covered for the six-minute walk test was $240.08 \pm 110.42 \mathrm{~m}$. The distance covered in the two-minute walk test had very strong correlation $(\mathrm{r}=0.9, p<0.01)$ with the distance covered in the six-minute walk test, indicating high construct validity of the two-minute walk test in this population. In addition, the correlation between the two-minute walk test and GMFCS levels was $(\mathrm{r}=$ $0.5, p<0.01$ ), showing a negative moderate correlation.

This study explored the reliability and validity of the two-minute walk test among children and adolescents with CP. This study demonstrated that the two-minute walk test had excellent test-retest reliability among the overall sample size. This study also showed excellent construct validity between the two- and six-minute walk tests.

The results from this study agreed with the outcomes from the previous study in children with and without neuromuscular conditions [16]. We conducted this study based on the recommendation of Pin and Choi [16], who suggested that the establishment of psychometrics for the two-minute walk test for specific diagnosis (i.e., CP) was warranted. Therefore, our findings add to the knowledge of the current available evidence. Excellent test-retest reliability demonstrated consistency between the two assessments and between the individuals for the two-minute walk test in children and adolescents with CP in this study. Compared to other studies with different populations, such as people with lower limb amputation ICC $=0.83$ [25], adults with neurological impairment with $\mathrm{ICC}=0.97$ [26], patients with chronic obstruction pulmonary disorder with ICC $=0.99$ [15], and stroke survivors with ICC $=0.98$ [11], this study showed excellent to good reliability for the two-minute walk test.

The mean distance in children with GMFCS level III was more consistent in comparison with those with GMFCS levels I and II during the second assessment; however, this did not reach to a significant level. This might be due to the fact that the participants with GMFCS levels I and II were more high functioning and able to walk faster compared to those with GMFCS level III.

The $\mathrm{MDC}_{95}$ was calculated as $12.7 \mathrm{~m}$, indicating that a child with $\mathrm{CP}$ had to have a greater than 12.7 $\mathrm{m}$ change to reflect a true change in walking performance, as measured by the two-minute walk test. This result is slightly lower than the reported $16.6 \mathrm{~m} \mathrm{MDC}_{90}$ on the two-minute walk test in children with neuromuscular impairments [16]. The difference might be due to the fact that our study focused on specific diagnosis (i.e., CP), whereas the study by Pin and Choi [16] included $>50 \%$ of non-CP population (i.e., neuromuscular conditions). Individuals with $\mathrm{CP}$ have difficulty walking due to spasticity, muscle weakness, or contracture, and these are not the main features of other conditions such as Down syndrome and muscular dystrophy as included in previous study.

This study also showed that the two- and six-minute walk tests had a strong significant correlation. In addition, the two-minute walk test was negatively correlated with the GMFCS levels. Participants with higher GMFCS levels walk slower and have less walking endurance; hence, this result is not surprising in indicating that the higher the GMFCS level, the lower the distance covered in two minutes. A previous study reported a high correlation between two and six-minute walk tests among healthy children and adults aged 3 - 85 years [27].

A testing interval of two weeks was chosen to decrease the learning effect that can occur with repeated testing and to avoid the effect of intervention on the walking performance. However, there is a possibility that, because of the variability of walking performance, i.e., the gait speed of high-functioning CP (GMFCS level I), the participants may have walked faster during the retest. In addition, the walk tests followed the same order for all participants; therefore, the results may have had bias, as no counterbalance of administration of the tests took place. While it is impossible to control the activities, we ensured that the participants did not go for any physiotherapy session in between the two assessments. The wide age range of the study population, i.e., children and adolescents, might have influenced the results of walking performance, as they might have had different activities with regards to participation at school and home. Further study with a larger sample is suggested to confirm the GMFCS subgroups reliability and MDCs, although small sample sizes in neuropediatric studies are common [28]. It is also recommended to establish the minimal clinically important differences in CP subgroups classified with GMFCS levels I, II, and III for future research. 
http://wjst.wu.ac.th

\section{Conclusions}

This is the first study to report the test-retest reliability and construct validity of the two-minute walk test for children and adolescents with CP and within GMFCS levels I-III. In summary, the findings show that the two-minute walk test demonstrated excellent test-retest reliability and high construct validity to measure walking performance in children and adolescents with CP. Future research is needed to determine MDCs and minimally clinical important changes in young people with $\mathrm{CP}$ across the GMFCS levels with a larger sample size.

\section{Acknowledgements}

The authors would like to thank all the participants and parents for participating in this study.

\section{References}

[1] P Rosenbaum, N Paneth, A Leviton, M Goldstein, M Bax and D Damiano. A report: The definition and classification of cerebral palsy April 2006. Dev. Med. Child. Neurol. Suppl. 2007; 109, 8-14.

[2] SH Zainah, LC Ong, A Sofiah, BK Poh and IH Hussain. Determinants of linear growth in Malaysian children with cerebral palsy. J. Paediatr. Child. Health 2001; 37, 376-81.

[3] LC Ong, NY Boo and V Chandran. Predictors of neurodevelopmental outcome of Malaysian very low birthweight children at 4 years of age. J. Paediatr. Child. Health 2001; 37, 363-8.

[4] TD Sanger, MR Delgado, D Gaebler-Spira, M Hallett and JW Mink. Classification and definition of disorders causing hypertonia in childhood. Pediatrics 2003; 111, e89-e97.

[5] TD Sanger, D Chen, MR Delgado, D Gaebler-Spira, M Hallett and JW Mink. Definition and classification of negative motor signs in childhood. Pediatrics 2006; 118, 2159-67.

[6] O Htwe, S Lloyd, MH Ng, A Richard, A Rashid, A Halim, AS Naicker and S Ibrahim. Urine hydroxyproline correlates with progression of spasticity in cerebral palsy. Eur. J. Gen. Med. 2018; 15, 1-9.

[7] N Z Zulkapli, N Z Saat and S Kamaralzaman. Postural control influence on upper extremity function among children with cerebral palsy: a literature review. Malay. J. Health Sci. 2016; 14, 1121.

[8] SW Õunpuu, G Gorton, A Bagley, MS -Williamson, S Hassani, B Johnson and D Oeffinger. Variation in kinematic and spatiotemporal gait parameters by Gross Motor Function Classification System level in children and adolescents with cerebral palsy. Dev. Med. Child. Neurol. 2015; 57, 955-62.

[9] E Wood and P Rosenbaum. The gross motor function classification system for cerebral palsy: A study of reliability and stability over time. Dev. Med. Child. Neurol. 2000; 42, 292-6.

[10] D Gijbels, BO Eijndel and P Feys. Comparison of the 2-and 6-minute walk test in multiple sclerosis. Mult. Scler. 2011; 17, 1269-72.

[11] M Kosak and T Smith. Comparison of the 2-, 6-, and 12-minute walk tests in patients with stroke. $J$. Rehabil. Res. Dev. 2005; 42, 103-7.

[12] A Zanudin, TH Mercer, KC Jagadamma and MVD Linden. Psychometric properties of measures of gait quality and walking performance in young people with Cerebral Palsy: A systematic review. Gait Posture 2017; 58, 30-40.

[13] AN Leunkeu, RJ Shephard and S Ahmaidi. Six-minute walk test in children with cerebral palsy gross motor function classification system levels I and II: Reproducibility, validity, and training effects. Arch. Phys. Med. Rehabil. 2012; 93, 2333-9.

[14] P Thompson, T Beath, J Bell, G Jacobson, T Phair, NM Salbach and FV Wright. Test-retest reliability of the 10-metre fast walk test and 6-minute walk test in ambulatory school-aged children with cerebral palsy. Dev. Med. Child. Neurol. 2008; 50, 370-6.

[15] AS Leung, KK Chan, K Sykes and KS Chan. Reliability, validity and responsiveness of a 2-min walk test to assess exercise capacity of COPD patients. Chest 2006; 130, 119-25. 
http://wjst.wu.ac.th

[16] TW Pin and HL Choi. Reliability, validity and norms of the 2-min walk test in children with and without neuromuscular disorders aged 6-12. Disabil. Rehabil. 2018; 40, 1266-72.

[17] TW Pin. Psychometric properties of two-minute walk test: A systemic review. Arch. Phys. Med. Rehabil. 2014; 9, 1759-75.

[18] ATS Committee on Proficiency Standards for Clinical Pulmonary Function Laboratories. ATS statement: Guidelines for the six-minute walk test. Am. J. Respir. Crit. Care Med. 2002; 166, 111-7.

[19] G Borg. Ratings of perceived exertion and heart rates during short-term cycle exercise and their use in a new cycling strength test. Int. J. Sports Med. 1982; 3, 153-8.

[20] P Shrout and JL Fleiss. Intraclass correlations: Uses in assessing rater reliability. Psychol. Bull. $1979 ; 86,420-8$.

[21] TK Koo and MY Li. A guideline of selecting and reporting intraclass correlation coefficients for reliability research. J. Chiropr. Med. 2016; 15, 155-63.

[22] HD Vet, CB Terwee, LB Mokkink and DL Knol. Measurement in medicine: A practical guide. University Press, Cambridge, UK, 2011.

[23] EM Andresen. Criteria for assessing the tools of disability outcomes research. Arch. Phys. Med. Rehabil. 2000; 81, S15-S20.

[24] JM Bland and D Altman. Statistical methods for assessing agreement between two methods of clinical measurement. Lancet 1986; 327, 307-10.

[25] L Resnik and LM Borgia. Reliability of outcome measures for people with lower-limb amputations: Distinguishing true change from statistical error. Phys. Ther. 2011; 91, 555-65.

[26] P Rossier and DT Wade. Validity and reliability comparison of 4 mobility measures in patients presenting with neurologic impairment. Arch. Phys. Med. Rehabil. 2001; 82, 9-13.

[27] RW Bohannon, D Bubela, S Magasi, H McCreath, YC Wang, D Reuben, WZ Rhymer and R Gershon. Comparison of walking performance over the first 2 minutes and the full 6 minutes of the Six-Minute Walk Test. BMC Res. Notes 2014; 7, 269.

[28] C Ammann-Reiffer, CH Bastiaenen, RAD Bie and HJV Hedel. Measurement properties of gaitrelated outcomes in youth with neuromuscular diagnoses: a systematic review. Phys. Ther. 2014; 94, 1067-82. 